$11-2017$

\title{
Hybrid Extended Kalman Filtering and Noise Statistics Optimization for Produce Wash State Estimation
}

\author{
Vahid Azimi \\ Georgia Institute of Technology \\ Daniel Munther \\ Cleveland State University, d.munther@csuohio.edu \\ Seyed Abolfazl Fakoorian \\ Cleveland State University \\ Thang Tien Nguyen \\ Cleveland State University \\ Daniel J. Simon \\ Cleveland State University, d.j.simon@csuohio.edu
}

Follow this and additional works at: https://engagedscholarship.csuohio.edu/scimath_facpub

Part of the Food Microbiology Commons, and the Mathematics Commons

How does access to this work benefit you? Let us know!

\section{Repository Citation}

Azimi, Vahid; Munther, Daniel; Fakoorian, Seyed Abolfazl; Nguyen, Thang Tien; and Simon, Daniel J., "Hybrid Extended Kalman Filtering and Noise Statistics Optimization for Produce Wash State Estimation" (2017). Mathematics Faculty Publications. 315.

https://engagedscholarship.csuohio.edu/scimath_facpub/315

This Article is brought to you for free and open access by the Mathematics and Statistics Department at EngagedScholarship@CSU. It has been accepted for inclusion in Mathematics Faculty Publications by an authorized administrator of EngagedScholarship@CSU. For more information, please contact library.es@csuohio.edu. 


\title{
Hybrid extended Kalman filtering and noise statistics optimization for produce wash state estimation
}

\author{
Vahid Azimi, Daniel Munther, Seyed Abolfazl Fakoorian, Thang Tien Nguyen, Dan Simon
}

\author{
A R T I C L E I N F O \\ Article history: \\ Received 1 October 2016 \\ Received in revised form \\ 19 May 2017 \\ Accepted 24 May 2017 \\ Available online 29 May 2017 \\ Keywords: \\ Produce wash \\ Kalman filter \\ Particle swarm optimization
}

\section{Introduction}

Fresh fruits and leafy green vegetables are an essential part of a healthy diet. However, produce carries a risk of pathogen contamination, which may result in food-borne illness (Lynch et al., 2009; Park et al., 2012; Sivapalasingam et al., 2004). Increased globalization and complexity of the supply chain indicates the need for sophisticated methods of monitoring to ensure fresh produce safety (Beuchat et al., 2004; Lynch et al., 2009). To address these needs, particularly at the processing juncture, recent years have witnessed numerous studies in fresh-cut produce washing (Barrera et al., 2012; Beuchat et al., 2004; Gil et al., 2009; Luo et al., 2011, 2012; Shen et al., 2013; Van Haute et al., 2013). In terms of chlorine sanitization, (Beuchat et al., 2004; Gómez-López et al., 2014;
Luo et al., 2011; Shen et al., 2013), studies have shown that the residual FC concentration in conjunction with sufficient contact time are essential factors for controlling pathogen inactivation and preventing cross-contamination. However, despite widespread use of chlorine, the underlying mechanisms that govern pathogen and FC dynamics during rapidly changing wash water conditions are not completely understood. One reason for this is that the relationships between washing control parameters and pathogen levels in wash water and on produce have only been described via experimental and correlative approaches or by risk models that are difficult to parameterize accurately (Barrera et al., 2012; Beuchat et al., 2004; Chen and Hung, 2016; Gómez-López et al., 2014; Luo et al., 2011; Pirovani et al., 2001; Rodríguez et al., 2011; Shen et al., 2013; Van Haute et al., 2013). While these results are important and have clear value, they have limited use for making precise predictions or elucidating real-time corrective measures.

To address such issues, an HEKF for state estimation of a produce 


\begin{tabular}{|ll|}
\hline Abbreviations \\
COD & chemical oxygen demand \\
CSH & concentrated sodium hypochlorite \\
PC & E. coli concentration \\
P & E. coli level \\
FC & free chlorine \\
HEKF & hybrid extended Kalman filter \\
MPN & most probable number \\
PSO & particle swarm optimization \\
RMSE & root mean square error \\
ORP & oxidation reduction potential \\
\hline
\end{tabular}

wash system is designed based on pilot-plant experiments (Luo et al., 2012), a mathematical model (Munther et al., 2015), and noise statistics optimization using PSO. State estimation has not been applied to produce washing prior to this research, but will soon be an essential component of wash systems for the following reasons: (1) state feedback controllers require accurate estimates of the system states; (2) real-time measurements (for instance, of surrogate water quality parameters such as ORP, turbidity, total dissolved solids, etc.) that inform chlorine control contain errors due to inaccurate sensors; (3) sensors may be expensive and prone to failure; and (4) there are time delays between the sensor measurements and their availability for real-time control.

State-of-the-art chlorine control relies on feedback informed by ORP and pH measurements in the wash water (Zhou et al., 2014). While such online systems provide better control than manual dosing, the most crucial issue is that chlorine dosing based on ORP measurements is limited since it does not exploit dynamic system information. Furthermore, in light of rapidly changing organic loads in the wash water, correlations used to link ORP to dosing needs may not be dynamic enough to maintain a desired level of FC.

This paper takes the state-of-the-art to the next level with state estimation of a dynamic system model that captures the fundamental kinetics of wash water chemistry and pathogen dynamics (Munther et al., 2015) and that is informed by discrete-time measurements (Luo et al., 2012) during a commercial wash process. This approach provides a predictive capacity beyond what correlative approaches can offer and is a first step toward improved control of FC. Although FC control is not the focus of this paper, the state estimation presented here will enable future research in statebased control of FC, pathogen concentration in the water, and pathogen on the lettuce, all while minimizing the FC input.

In general, state estimation algorithms can exploit real-time measurements to provide optimal estimates and predictions of the system states (Simon, 2006; Fakoorian et al., 2016, 2017). For example, the states of general constrained nonlinear systems have been estimated using moving horizon estimation and Carleman linearization (Hashemian and Armaou, 2015, 2016). The EKF continues to be the most popular state estimation technique for nonlinear systems (Bellantoni and Dodge, 1967). In addition, HEKF performance is heavily dependent on the noise model; the estimator performance may degrade or even diverge if the noise is incorrectly modeled. Various approaches have been used to identify unknown noise characteristics to achieve accurate estimation. In Kontoroupi and Smyth (2016), an adaptive method for online process and measurement noise identification during joint state and parameter estimation of nonlinear systems was presented. Identification of the noise covariance matrices using an autocovariance least-squares method for linear systems was implemented in (Kost et al., 2015).

In this research, system noise is identified from experimental system measurements, which is then used in the system simulation. However, since the system and the estimator are nonlinear, noise covariance matrices in the estimator are different than the true covariance matrices. Therefore, PSO is used to optimize the covariance matrices to use in the estimator to obtain the best possible estimation performance. In summary, the system noise is first identified offline using experimental data captured during a wash process, and the covariance matrices are then optimized offline using PSO for use in the real-time filter.

In Section 2, the experimental procedures that were used to generate the data and subsequently used to inform the chlorine/ cross-contamination dynamic model and identify the system process noise are described. In Section 3, the HEKF and the noise statistics optimization framework are presented, along with the PSO algorithm for noise statistics optimization for the HEKF. The proposed approach uses the fact that in an optimal linear Kalman filter, the measurement innovations is zero-mean white noise with a known covariance; the noise model can thus be tuned to achieve theoretically desired HEKF behavior. In Section 4, simulation results are presented which show that the system provides good estimation of the states. Specifically, in Section 4.2 PSO reduces the estimation cost function by $34 \%$ and drives the innovations as close as possible to zero-mean white noise with the desired covariance. In Section 4.3, the simulated state estimation results have an RMSE of $8.24 \mathrm{mg} / \mathrm{L}$ for COD, $0.09 \mathrm{mg} / \mathrm{L}$ for FC concentration, $0.19 \mathrm{MPN} / \mathrm{ml}$ for PC in the water wash, and $0.04 \mathrm{MPN} / \mathrm{g}$ for P on the lettuce. Sensitivity analysis to examine the robustness of the state estimator is also presented in Section 4. As discussed in Section 4.5, the greatest sensitivity is reflected in the estimated COD relative to the COD increase rate parameter, which is 73 ; that is, an error of one unit in the modeled COD increase rate results in a 73 -fold increase in COD estimation error. The lowest sensitivity is the that of the estimated lettuce P to the natural decay rate of the FC, which is 0.26 . Finally, in Section 5 concluding remarks and future work are discussed.

\section{Dynamic model and system process noise}

\subsection{Experimental procedure}

The experimental study (Luo et al., 2012) took place in a commercial pilot plant (New Leaf Food Safety Solutions, LLC, in Salinas, California). The setup consisted of a commercial double wash system, each tank with approximately 3200 L capacity and equipped with rotating screens to ensure produce submersion, and air pumps to create turbulence in the wash water. Baby spinach leaves were spray inoculated with $E$. coli 0157:H7 strain ATCC 700728 (average level of $2 \times 10^{5} \mathrm{CFU} / \mathrm{g}$ of spinach) prior to washing. Before each test, approximately $22.5 \mathrm{~kg}$ per tote of pre-cored iceberg lettuce heads were shredded to $0.6 \mathrm{~cm}$-thick shreds using an Urschel slicer (Urschel Laboratories, Inc., Valparaiso, Indiana).

To simulate the cross-contamination process, inoculated baby spinach leaves were continuously placed parallel to (but not touching) shredded iceberg lettuce on a conveyor belt emptying into the primary wash tank at a fixed rate. The spinach to lettuce ratio was $0.2 \%$. While the process water was continuously screened and re-circulated, produce spent an average of $26 \mathrm{~s}$ in each tank. The $\mathrm{pH}$ in the wash water was maintained at 6.5 using citric acid. Each test run involved three consecutive 12-min segments, simulating continuous processing with a periodic FC dosing scheme. At the start of each segment, concentrated (12.5\%) sodium hypochlorite (BCS Chemicals, Redwood City, California) was added to the primary wash tank to achieve a desired equilibrium level of FC (see Figs. 1 and 3 in Luo et al., 2012). 
Note that because preliminary experiments suggested that FC levels in the secondary tank were relatively stable, sample collection only occurred in the primary tank. Water samples from the upper water level of the primary tank and lettuce samples on the conveyor belt were collected before each run and then water samples and lettuce samples from the upper water level of the primary tank (between the rotating screen and end of the tank) were collected every 2 min during washing (see Fig. 1 in Luo et al., 2012). FC was measured immediately based on a DPD method (N, $\mathrm{N}$-Diethyl-p-Phenylenediamine) using a chlorine photometer (CP25, HF Scientific Inc., Ft. Myers, Florida) with an accuracy of $0.01 \mathrm{ppm}$ for $0-6 \mathrm{ppm}$ and $0.1 \mathrm{ppm}$ for $6-10 \mathrm{ppm}$. $\mathrm{pH}$ and turbidity were measured on-site using a digital $\mathrm{pH}$ meter and a digital turbidity meter (Aquafast, Thermo Orion, Beverly, Massachusetts), and COD was determined by using a reactor digestion method 10236 (Hach, 2002; Luo, 2007). The COD measurements are accurate up to $2 \mathrm{ppm}$ for $150-1500 \mathrm{ppm}$. As shredded lettuce continuously entered the wash system (45 kg/min), COD increased linearly from an initial value of $300 \mathrm{mg} / \mathrm{l}$ to $1600 \mathrm{mg} / \mathrm{l}$ at $36 \mathrm{~min}$ (see Fig. 2 in Luo et al., 2012). Furthermore, the E. coli 0157:H7 levels on the lettuce and in water samples were determined using a modified MPN method as described in (Luo et al., 2011, 2012; Nou and Luo, 2010). Accuracy for measured pathogen levels was $0.038 \mathrm{MPN} / \mathrm{g}$ on lettuce and $0.38 \mathrm{MPN} / \mathrm{ml}$ in the water.

\subsection{Chlorine/cross-contamination dynamic model}

A dynamic model for the commercial double wash system is illustrated in Fig. 1 (Luo et al., 2012; Munther et al., 2015). As mentioned in Subsection 2.1, since preliminary experiments indicated that FC levels in the secondary tank were relatively stable, sample collection occurred only in the primary tank (Luo et al., 2012). The chlorine and cross-contamination dynamics of the primary tank can be modeled as follows (Munther et al., 2015):

$\underline{\dot{x}}=f+g u+w_{s}(t)$

$f(\underline{x})=\left[\begin{array}{c}K_{0} \\ -\gamma_{c} x_{2}-\beta_{c} x_{1} x_{2} \\ \beta_{w s}-\beta_{l w}\left(\frac{L}{V}\right) x_{3}-\alpha x_{2} x_{3} \\ \beta_{l w} x_{3}-\alpha x_{2} x_{4}-C_{l} x_{4}\end{array}\right] \quad g=\left[\begin{array}{l}0 \\ 1 \\ 0 \\ 0\end{array}\right]$

$y_{k}=x_{2 k}+v_{s_{k}}$

In the vector $f$, the first two equations represent the water chemistry dynamics in the wash tank, and the last two equations represent the pathogen contamination dynamics in the wash water and on produce in the tank. $\underline{x}=\left[\begin{array}{llll}x_{1} & x_{2} & x_{3} & x_{4}\end{array}\right]^{T}=$ $\left[\begin{array}{llll}O & C & X_{W} & X_{L}\end{array}\right]^{T}$ denotes the states of the system; $O(\mathrm{mg} / \mathrm{L})$ is COD in the water wash; $C(\mathrm{mg} / \mathrm{L})$ represents $\mathrm{FC}$ concentration; $X_{W}(\mathrm{MPN} / \mathrm{ml})$ is the PC in the water wash; $X_{L}(\mathrm{MPN} / \mathrm{g})$ represents

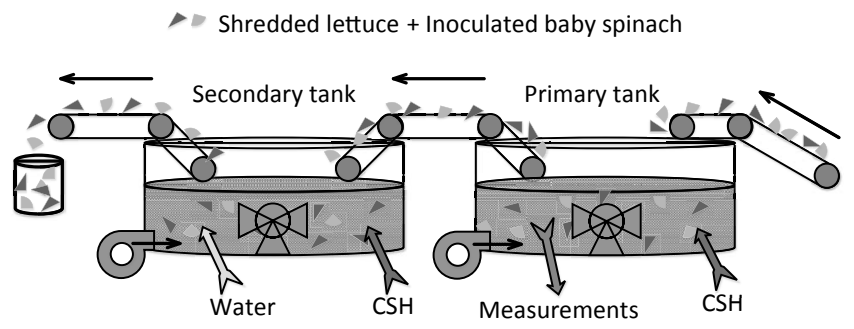

Fig. 1. Commercial double wash system.

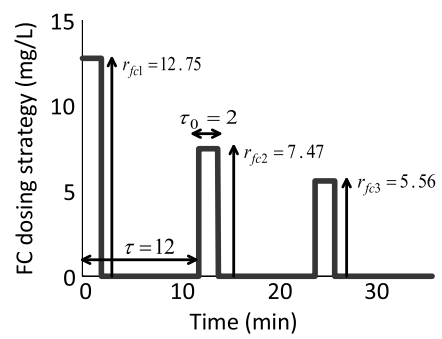

Fig. 2. FC dosing strategy. The magnitude of the FC doses $r_{f c_{i}}, i=1,2,3$ are calculated in (Munther et al., 2015) with a least-squares fit to the model of Eq. (1). The dosing width $\tau_{0}$ and the FC dosing period $\tau$ are from (Luo et al., 2012).

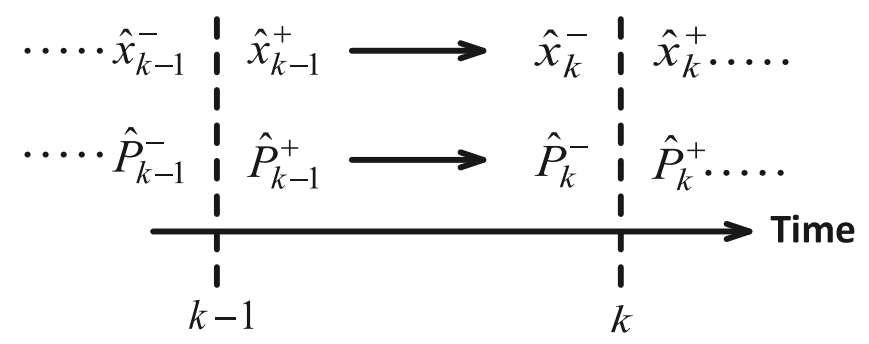

Fig. 3. Prior and posterior estimates and covariances.

the P on the lettuce; $u(\mathrm{mg} / \mathrm{l} \mathrm{min})$ represents the $\mathrm{FC}$ injection rate to the wash tank (see Fig. 2); $y_{k}$ represents the discrete-time measurement, which is FC concentration; $w_{s}(t)$ represents the fourelement continuous-time white process noise vector of the system with $n \times n$ covariance $Q_{s} ; v_{s_{k}}$ represents the scalar discrete-time white measurement noise with $m \times m$ covariance $R_{s_{k}}$; and $n$ and $m$ are the number of states and measurements respectively.

When fresh-cut produce enters the wash system, the abrupt increase in organic matter from exudates results in a linear increase in COD (Fig. 2 in Luo et al., 2012). The increase of COD is quantified by $K_{0}$ (mg/l-min). In terms of FC decay, $\gamma_{c}(1 / \mathrm{min})$ indicates the natural decay rate of FC in water, and $\beta_{c}(1 / \mathrm{mg}-\mathrm{min})$ represents a type of second order rate constant quantifying depletion due to organics in the water (Deborde and Von Gunten, 2008; Munther et al., 2015). To compensate for the decrease in hypochlorous acid, the experimental procedure outlined in Section 2.1 is followed (Luo et al., 2012) and a periodic FC dosing scheme with fixed period $\tau=12$ minutes is used. Three doses are used with a fixed dosing width (duration) $\tau_{0}=2$ minutes, and the FC rate for each dose is denoted as $r_{f c_{i}}, i=1,2,3$ (Luo et al., 2011, 2012; Munther et al., 2015).

Fig. 2 illustrates the FC dosing strategy over the 36 min duration of the entire experiment. Water was recirculated during the experiment (Luo et al., 2012); however, to simplify the model, and also because of the lack of data for FC returning to the tank, this dynamic is not included in the chlorine equation. Also, while the equation for the FC dynamics does not explicitly account for chlorine breakpoint phenomena, the organic load/FC relationship in this context is implicitly captured in terms of the FC dose rates $r_{f c_{i}}$ and duration. That is, only experimental data during the dosing time can be relied on to quantify these dose rates (Luo et al., 2012). Future work will consider both experimentation and modeling in the context of continuous chlorine dosing to explicitly quantify the breakpoint dynamics.

\subsection{Covariance matrix of the system's process noise}

The measurement noise variance $R_{S_{k}}=1$ is used in the state estimator; this is because it is only the ratio of $Q_{s}$ to $R_{S_{k}}$ (not their absolute values) that determines estimation performance. $K_{0}$ and 
$\beta_{c}$ in Equation (1) are positive constants and $1 / C_{l}$ denotes the average dwell time for the lettuce in the wash tank. In the next section, an HEKF is presented to estimate the system states using the measurements and the system model. The system parameters are summarized in Table 1. Before implementing the HEKF, this section shows how experimental data is used to derive the discretetime process noise covariance $Q_{S_{d}}$ which corresponds to the continuous-time covariance $Q_{s}$. Equation (1) shows that the equivalent discrete-time process noise can be approximated as

$w_{s_{m}}=\frac{x_{\exp _{m+1}}-x_{\exp _{m}}-\left(f\left(x_{\exp _{m}}\right)+g u_{m}\right) \Delta t}{\Delta t}$

where $w_{s_{m}}$ represents a four-element discrete-time process noise vector for $m=1, \ldots, m_{\max }$, where that $m_{\max }$ is the number of experimental data points; $x_{\text {exp }}$ is the $m$-th experimental data point captured during the washing process (measurement $\Delta t=2 \mathrm{~min}$ ); $f\left(x_{\exp _{m}}\right)$ is the system dynamics in (1) evaluated at $x_{\text {exp }}$; and $u_{m}$ is the FC dosing strategy shown in Fig. 1 at sample index $m$. The covariance $Q_{s_{d}}$ is estimated as

$Q_{s_{d}}=\sum_{m=1}^{m_{\max }} \frac{w_{s_{m}}^{2}}{m_{\max }}$

The continuous-time process noise covariance $Q_{S}$ can then be obtained as follows (Simon, 2006, Chapter 8):

$Q_{s}=\frac{Q_{s_{d}}}{\Delta t}$

Nineteen measured experimental samples of chlorine are used during the wash process, so $m_{\max }=19$ in Eq. (3). The $4 \times 4$ covariance matrix of the system's process noise $Q_{s}$ is calculated from the experimental data using Eqs. (2)-(4) as

$Q_{s}=\operatorname{diag}\left(\begin{array}{llll}468 & 0.64 & 1.77 & 1.34\end{array}\right)$

\section{Hybrid extended Kalman filter and noise statistics optimization}

In this section, an HEKF is designed to estimate the system states of Eq. (1), and then PSO is used to optimize the noise statistics so that the HEKF is approximately optimal.

\subsection{Hybrid extended Kalman filter}

Consider a general continuous-time system with discrete time measurements (Simon, 2006):

$$
\begin{aligned}
& \dot{x}=f\left(x, u, w_{s}, t\right) \\
& y_{k}=h_{k}\left(x_{k}, v_{s_{k}}\right) \\
& w_{s}(t) \sim\left(0, Q_{s}\right) \\
& v_{s_{k}} \sim\left(0, R_{s_{k}}\right)
\end{aligned}
$$

$f($.$) and h_{k}($.$) represent the nonlinear system and measurement$ equations; and $y_{k}, x$, and $u$ are the system measurement, state, and input, respectively. The filter is initialized as follows:

$\widehat{x}_{0}^{+}=\mathrm{E}\left[x_{0}\right]$
$P_{0}^{+}=\mathrm{E}\left[\left(x_{0}-\widehat{x}_{0}^{+}\right)\left(x_{0}-\widehat{x}_{0}^{+}\right)^{T}\right]$

where $\mathrm{E}($.$) denotes the expected value operation, and P_{0}^{+}$is the covariance of the initial estimate. For each time index $k$, the state estimate and its covariance are integrated from time $(k-1)^{+}$to time $k^{-}$as follows (Simon, 2006):

$\dot{\widehat{x}}=f(\widehat{x}, u, 0, t)$

$\dot{P}=A P+P A^{T}+L Q_{f} L^{T}$

where $A$ and $L$ denote the partial derivatives of $f\left(x, u, w_{s}, t\right)$ with respect to $x$ and $w_{s}$ respectively, both evaluated at $\widehat{x}$; and $Q_{f}$ denotes the continuous-time process noise covariance used in the HEKF algorithm. Note that $Q_{f}$ is, in general, different from $Q_{s}$ because $Q_{s}$ is unknown in practice. Although $Q_{s}$ was identified in Section 2, it was identified only for one specific experiment. In general, $Q_{S}$ is unknown and so the HEKF needs to be implemented apart from any knowledge of $Q_{s}$.

Eq. (7) is integrated starting from time index $k-1$ with $\widehat{x}=\widehat{x}_{k-1}^{+}$ and $P=P_{k-1}^{+}$and ending at the next time index $k$ with $\widehat{x}=\widehat{x}_{k}^{-}$and $P=P_{k}^{-}$, where $\widehat{x}_{k}^{-}$and $P_{k}^{-}$are the prior estimate and covariance respectively. Fig. 3 provides a conceptual diagram of the prior and posterior estimates and covariances (Simon, 2006). The measurement $y_{k}$ is incorporated into the state estimate and estimation covariance at time $k$ as follows:

$$
\begin{aligned}
& K_{k}=P_{k}^{-} H_{k}^{T}\left(H_{k} P_{k}^{-} H_{k}^{T}+M_{k} R_{f_{k}} M_{k}^{T}\right)^{-1} \\
& \widehat{x}_{k}^{+}=\widehat{x}_{k}^{-}+K_{k}\left(y_{k}-h_{k}\left(\widehat{x}_{k}^{-}, 0, t_{k}\right)\right) \\
& P_{k}^{+}=\left(I-K_{k} H_{k}\right) P_{k}^{-}\left(I-K_{k} H_{k}\right)^{T}+K_{k} M_{k} R_{f_{k}} M_{k}^{T} K_{k}^{T}
\end{aligned}
$$

where $H_{k}$ and $M_{k}$ denote the partial derivative of $h_{k}\left(x_{k}, v_{s k}\right)$ with respect to $x_{k}$ and $v_{s k}$ respectively, both evaluated at $\widehat{x}_{k}^{-} ; K_{k}$ is the Kalman filter gain; $y_{k}$ denotes the measurement at time $k$; and $R_{f_{k}}$ denotes the discrete-time measurement noise covariance.

\subsection{Noise statistics optimization}

Now that the HEKF algorithm has been outlined, a method is proposed to optimize the noise statistics of the covariances that are used in the HEKF. Both measurement and process noise statistics are unknown, but the performance of the HEKF strongly depends on accurate noise models, so a noise model optimization method is derived using PSO. The process and measurement noises are assumed to be uncorrelated with diagonal covariance matrices.

The dynamics in Eq. (1) has four states, and FC concentration is the only measurement $(n=4, m=1)$, so the problem here is to tune the four diagonal elements of $Q_{f}$ and the single element of $R_{f_{k}}$.

Table 1

System parameters used in Eq. (1); see (Munther et al., 2015) for details.

\begin{tabular}{lll}
\hline Parameter & Description & Value (Units) \\
\hline$K_{0}$ & COD increase rate & $32.3(\mathrm{mg} /(\mathrm{L} \mathrm{min}))$ \\
$\gamma_{c}$ & Natural decay rate of FC & $1.7 \times 10^{-3}(1 / \mathrm{min})$ \\
$\beta_{c}$ & FC consumption rate & $5.38 \times 10^{-4}(\mathrm{~L} / \mathrm{mg} \mathrm{min})$ \\
$\beta_{w s}$ & Increase rate of the pathogen in the water & $1.95(\mathrm{MPN} /(\mathrm{ml} \mathrm{min}))$ \\
$\beta_{l w}$ & Pathogen binding rate (water to produce $)$ & $0.38(\mathrm{ml} /(\mathrm{g} \mathrm{min}))$ \\
$L$ & Amount of lettuce in the tank & $19526(\mathrm{~g})$ \\
$V$ & Volume of the tank & $3.2 \times 10^{6}(\mathrm{ml})$ \\
$C_{l}$ & Reciprocal of average wash time & $2.3(1 / \mathrm{min})$ \\
$\alpha$ & Interaction rate of the pathogen via FC & $0.5(1 /(\mathrm{mg} \mathrm{min}))$ \\
\hline
\end{tabular}


The innovations are used for optimization, and are defined as

$$
r_{k}=y_{k}-h_{k} \widehat{x}_{k}^{-}
$$

The innovations represent that part of the measurement that contains new information about the state. PSO is used to optimize the diagonal elements of $Q_{f}$ and $R_{f_{k}}$ so that the proposed HEKF is approximately optimal, which means the innovations satisfy three different conditions (Simon, 2006): (1) white noise, (2) zero mean, and (3) covariance equal to $H_{k} P_{k}^{-} H_{k}^{\mathrm{T}}+R_{s_{k}}$. Fig. 4 depicts the structure of the HEKF/noise statistics optimization system.

\subsubsection{Particle swarm optimization}

In this section, PSO is used to optimize the process noise statistics for the HEKF; that is, the diagonal elements of the covariance matrix $Q_{f}$ and the single element $R_{f_{k}}$ are tuned so that the innovations are approximately white noise and zero mean with a covariance of $H_{k} P_{k}^{-} H_{k}^{\mathrm{T}}+R_{s_{k}}$. For the first condition, the whiteness of the innovations, the lower and upper bounds of a $99 \%$ statistical confidence interval for the whiteness of the innovations are calculated. If the sample autocorrelation values are within the $99 \%$ confidence bounds, it is concluded that the innovations are white. The RMSE difference between the measured and estimated chlorine is also incorporated in the cost function as a heuristic to ensure reasonable estimation performance. Therefore, four individual cost function components are combined with weighting coefficients to obtain the overall PSO cost function:

$$
\begin{aligned}
& \operatorname{Cost}_{1}=\frac{1}{g_{\max }} \sum_{g=1}^{g_{\max }}\left|R_{g}\right| \\
& \operatorname{Cost}_{2}=\frac{1}{N} \sum_{k=1}^{N}\left|r_{k}\right| \\
& \operatorname{Cost}_{3}=\frac{1}{N} \sum_{k=1}^{N}\left|r_{k}^{2}-\left(H_{k} P_{k}^{-} H_{k}^{\mathrm{T}}+R_{S_{k}}\right)\right| \\
& \operatorname{Cost}_{T}=\sum_{e=1}^{3} \zeta_{e} \operatorname{Cost}_{e}+\zeta_{4} R M S E_{y}
\end{aligned}
$$

where $R_{g}=\frac{1}{N-g} \frac{\sum_{i=1}^{N-g}\left(r_{i}-\bar{r}\right)\left(r_{i+g}-\bar{r}\right)}{r^{2} \sigma^{2}}$ is the normalized autocorrelation of innovation samples ( $N$ is the number of FC measurement samples); $\bar{r}$ and $\sigma_{r}^{2}$ denote the sample mean and variance of the innovations; $g=1, \ldots, g_{\max }$ denotes the time lag index of the autocorrelation sample values, where $g_{\max }<N$ is the maximum lag; $R M S E_{y}$ denotes the root mean square error between the measured and estimated chlorine data; and $\zeta_{e}(e=1,2,3,4)$ are weighting coefficients that indicate the relative importance of each individual cost function component. PSO uses Eq. (10) to adjust the diagonal

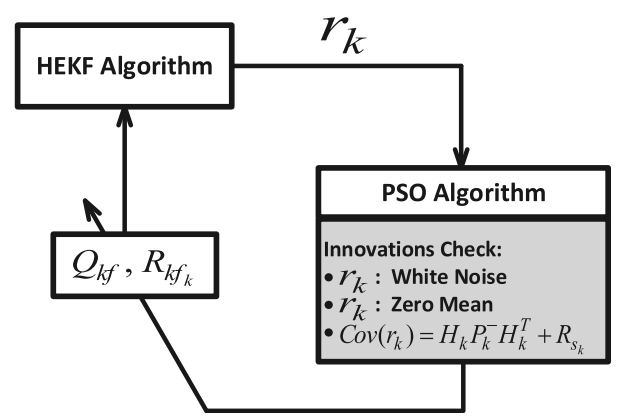

Fig. 4. Proposed structure for the HEKF and noise identification. elements of $Q_{f}$ and $R_{f_{k}}$ so that the innovations satisfy their theoretically desired properties.

\subsubsection{Overview of PSO}

PSO is a population-based optimization algorithm that is motivated by the social behavior of animals (Kennedy and Eberhart, 1995; Eberhart and Kennedy, 1995). PSO shares information between candidate solutions to find an optimal solution using a blend of deterministic and random operations. Compared to other evolutionary computation techniques, PSO is easy to implement, has only a few tuning parameters, and is computationally efficient. PSO is also attractive due to its ability to find the global solution to optimization problems.

These features motivate us to use PSO to find the optimal individual in the population of candidate solutions and to minimize the cost function in Eq. (10). PSO begins by initializing a population (swarm) of individuals that are assigned random initial positions and zero velocities; the position of each individual represents a candidate solution, which includes diagonal elements of the process noise covariance. Their velocities represent the directions that they move through the search space. Each individual's velocity is updated using the difference between its best position in the past and its current position, and the difference between the best position of its neighbors and its current position. The positions of the individuals are then updated using their velocities. A maximum number of iterations (that is, generations) is defined as the stopping criteria. The velocity update equation is presented as follows (Simon, 2013):

$$
\begin{aligned}
& \nu_{i}(\kappa+1)=Y \nu_{i}(\kappa)+C_{1}\left(b_{i}(\kappa)-z_{i}(\kappa)\right)+C_{2}\left(s_{i}(\kappa)-z_{i}(\kappa)\right), i=1, \ldots, l \\
& Y=K Y_{d}, C_{1}=K \theta_{1}, \quad C_{2}=K \theta_{2}
\end{aligned}
$$

Eq. (11) is a $D$-element vector operation, where $D$ is the dimension of the optimization problem; $\kappa=1, \ldots, \kappa_{\max }$ where $\kappa_{\max }$ is the maximum number of generations; $i=1, \ldots, l$ where $l$ is the number of particles (individuals); $z_{i}(\kappa), \nu_{i}(\kappa)$, and $b_{i}(\kappa)$ are the position, velocity, and prior best position of the $i^{\text {th }}$ particle at the $\kappa^{\text {th }}$ generation; $s_{i}(\kappa)$ is the best position of the $i^{\text {th }}$ neighborhood at the $\kappa^{\text {th }}$ generation; $\nu_{i}(\kappa+1)$ is the velocity of the $i^{\text {th }}$ particle at the $(\kappa+1)^{s t}$ generation; $\theta_{1}$ and $\theta_{2}$ are the cognition learning rate and the social learning rate respectively, which are random numbers uniformly distributed in $\left[0, \theta_{1, \max }\right]$ and $\left[0, \theta_{2, \max }\right]$ respectively each generation, where $\theta_{1, \max }$ and $\theta_{2, \max }$ are tuning parameters; $Y$ is the inertia weight, which decreases from 0.9 in the first generation to 0.1 in the last one; $Y_{d}$ is the damping ratio of $Y$; and $C_{1}$ and $C_{2}$ are velocity update coefficients. $K$ is the constriction coefficient and can be set as follows to ensure stability (Simon, 2013):

$$
K<\frac{2}{\theta_{1, \text { max }}+\theta_{2, \text { max }}-2+\sqrt{\left(\theta_{1, \text { max }}+\theta_{2, \text { max }}\right)^{2}-4\left(\theta_{1, \text { max }}+\theta_{2, \text { max }}\right)}}
$$

Velocity limiting is applied to the $i^{\text {th }}$ particle as follows:

$$
\nu_{i j}(\kappa+1) \leftarrow\left\{\begin{array}{cc}
\nu_{i j}(\kappa+1) & \left|\nu_{i j}(\kappa+1)\right| \leq \nu_{j}^{\max } \\
\nu_{j}^{\max } \operatorname{sgn}\left(\nu_{i j}(\kappa+1)\right), & \left|\nu_{i j}(\kappa+1)\right|>\nu_{j}^{\max }
\end{array}\right.
$$

where $j=1, \ldots, D$, and $\nu_{j}^{\max }$ is the maximum velocity, which is defined as

$\nu_{j}^{\max }=\sigma\left(z_{j}^{\max }-z_{j}^{\min }\right)$

where $z_{j}^{\max }$ and $z_{j}^{\min }$ are the minimum and maximum values of the $j^{\text {th }}$ dimension of the search domain; and $\sigma$ is a scale factor. After the 
velocity update of Eqs. (11) - (15), the position update and limiting are performed as follows:

$z_{i}(\kappa+1)=\nu_{i}(\kappa+1)+z_{i}(\kappa)$

$z_{i j}(\kappa+1) \leftarrow \min \left(z_{i j}(\kappa+1), z_{j}^{\max }\right)$

$z_{i j}(\kappa+1) \leftarrow \max \left(z_{i j}(\kappa+1), z_{j}^{\text {min }}\right)$

where Eq. (15) is a $D$-element vector operation, and $z_{i}(\kappa+1)$ is the position of the $i^{\text {th }}$ particle at the $(\kappa+1)^{s t}$ generation.

\section{Simulation results}

\subsection{Initialization}

PSO is used to identify the process and measurement noise statistics for the HEKF so the innovations form a zero-mean white noise process with covariance $H_{k} P_{k}^{-} H_{k}^{\mathrm{T}}+R_{s_{k}}$. The initial state of the system (Luo et al., 2012) is $\underline{x}_{0}^{T}=\left[\begin{array}{llll}300 & 0 & 0 & 0\end{array}\right]$, and the wash period is $36 \mathrm{~min}$. The initial covariance of the estimation error $P_{0}=10 I_{4 \times 4}$, and the maximum number of time lags $g_{\max }=5$, which were manually chosen to provide good performance.

Table 2 presents the parameters that are used in the PSO algorithm and the cost function of Eq. (10). The PSO parameters in Table 2 are chosen to provide good performance and fast convergence. Note that a larger number of iterations $\kappa_{\max }$ and a larger population size $l$ could be considered to possibly obtain better performance, but better performance would come at the expense of more computational time. The cost function coefficients $\zeta_{e}$ were chosen to make the weighted values of the components Cost $_{1}$, Cost $_{2}$, Cost $_{3}$, and RMSE $E_{y}$ of the same order of magnitude.

\subsection{Process and measurement noises of HEKF algorithm}

The continuous-time process noise covariance $Q_{s}$ was computed in Section 2. The standard deviation of the simulation process noise is the square root of the covariance matrix $S D_{Q s}=\sqrt{Q_{S}}=\operatorname{diag}\left(\begin{array}{llll}21.65 & 0.79 & 1.33 & 1.15\end{array}\right)$ and the standard deviation of the measurement noise is $S D_{R_{s_{k}}}=\sqrt{R_{S_{k}}}=1$. The PSO search domain of the standard deviation of the HEKF process and measurement noises needs to be bounded. The search domains are set to comfortably include the standard deviations of the simulation noise, which are the square roots of the system simulation noise covariance matrices $Q_{s}$ and $R_{S_{k}}$. The search domains are chosen as

$s q_{11} \in[0,40], s q_{22} \in[0,2], s q_{33} \in[0,3], s q_{44} \in\left[\begin{array}{ll}0, & 3\end{array}\right]$ $S D_{R_{f_{k}}} \in[0.1,2]$

Table 2

PSO parameters.

\begin{tabular}{lll}
\hline Parameter & Description & Value \\
\hline$D$ & Optimization problem dimension & 5 \\
$K_{\max }$ & Number of iterations & 20 \\
$l$ & Population size & 20 \\
$\theta_{1, \max }$ & Maximum rate for cognition learning & 2.05 \\
$\theta_{2, \max }$ & Maximum rate for social learning & 2.05 \\
$r_{d}$ & Damping ratio for inertia rate & 0.9 \\
$\sigma$ & Scale factor & 0.1 \\
$\zeta_{1}$ & Cost $_{1}$ coefficient & 100 \\
$\zeta_{2}$ & Cost $_{2}$ coefficient & 1 \\
$\zeta_{3}$ & Cost $_{3}$ coefficient & 1 \\
$\zeta_{4}$ & RMSE $_{y}$ coefficient & 1 \\
$g_{\max }$ & Maximum number of lag & 5 \\
$N$ & Number of innovations samples & 3601 \\
\hline
\end{tabular}

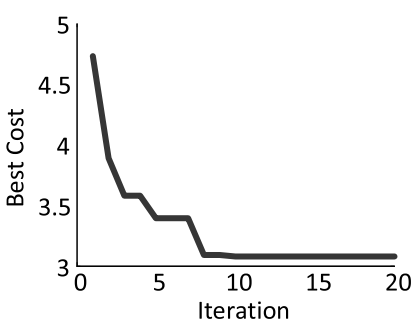

Fig. 5. PSO performance for identifying the process and measurement noise covariances.

PSO decreases $\operatorname{Cost}_{T}$ of Eq. (10) from 4.73 at the initial generation to 3.08 at the 20th generation, which means that the total cost improves by $34 \%$. Fig. 5 shows the best cost values of the PSO population over 20 generations. The best solution found by PSO is $S D_{Q f}=\operatorname{diag}\left(\begin{array}{llll}28.32 & 0.28 & 1.40 & 1.41\end{array}\right)$ and $S D_{R_{S_{k}}}=1.33$. The individual cost functions values from Eq. (7) for this solution are Cost $_{1}=0.008$, Cost $_{2}=0.79$, Cost $_{3}=1.38$, and $R M S E_{y}=0.98$.

\subsection{Verification of state estimation and noise statistics optimization}

Fig. 6 compares the states of the produce wash system with the estimated HEKF states for two situations: zero initial state estimation error $\left(\underline{x}_{0}^{T}=\left[\begin{array}{llll}300 & 0 & 0 & 0\end{array}\right]\right)$, and non-zero initial state estimation error $\left(\underline{x}_{0}^{T}=\left[\begin{array}{llll}350 & 10 & 5 & 0.5\end{array}\right]\right)$. Fig. 6 shows that with zero initial state estimation error the HEKF can accurately estimate the states with an RMSE of $8.24 \mathrm{mg} / \mathrm{L}$ for COD, $0.09 \mathrm{mg} / \mathrm{L}$ for FC, $0.19 \mathrm{MPN} / \mathrm{ml}$ for $X_{W}$, and $0.04 \mathrm{MPN} / \mathrm{g}$ for $X_{L}$. In the case of non-zero initial state estimation error the HEKF estimates the states with an RMSE of $18.29 \mathrm{mg} / \mathrm{L}$ for COD, $0.12 \mathrm{mg} / \mathrm{L}$ for FC, $0.63 \mathrm{MPN} / \mathrm{ml}$ for $X_{W}$, and $0.1 \mathrm{MPN} / \mathrm{g}$ for $X_{L}$. It is seen that the HEKF demonstrates good robustness against initial state estimation errors.

Fig. 7 shows the Kalman gains. The Kalman gain of the second state (FC concentration) converges to zero. This implies that sufficient knowledge from the measurements is eventually obtained so that additional ones do not provide any new information, so additional measurements are eventually ignored with respect to FC estimation updates. On the other hand, the Kalman gains of the first, third, and fourth states do not converge to zero, so the estimates of those states are more responsive to measurements. Note that the magnitude of the first Kalman gain is heuristically limited to 0.5 to obtain robust state estimation for COD.

Fig. 8 demonstrates the innovations of Eq. (9). Recall that the PSO algorithm optimizes the HEKF process and measurement noises so that the innovations are zero-mean white noise with covariance $H_{k} P_{k}^{-} H_{k}^{\mathrm{T}}+R_{S_{k}}$. The figure shows that the innovations appear to be approximately zero mean, and they also appear to be white, although the covariance cannot be easily identified from the figure.

Fig. 9 shows that the sample autocorrelation values of the innovations are bounded within the $99 \%$ confidence interval, except at zero time lag. It can be concluded that the innovations are approximately white.

\subsection{Monte Carlo simulation}

Monte Carlo simulation provides an additional tool to provide confidence in the state estimator. The sample standard deviation of the state estimation error $S_{e}$ is measured over multiple Monte Carlo simulations. The average standard deviation of the estimation error for the $j^{\text {th }}$ state is calculated over $N_{m c}$ Monte Carlo simulations: 

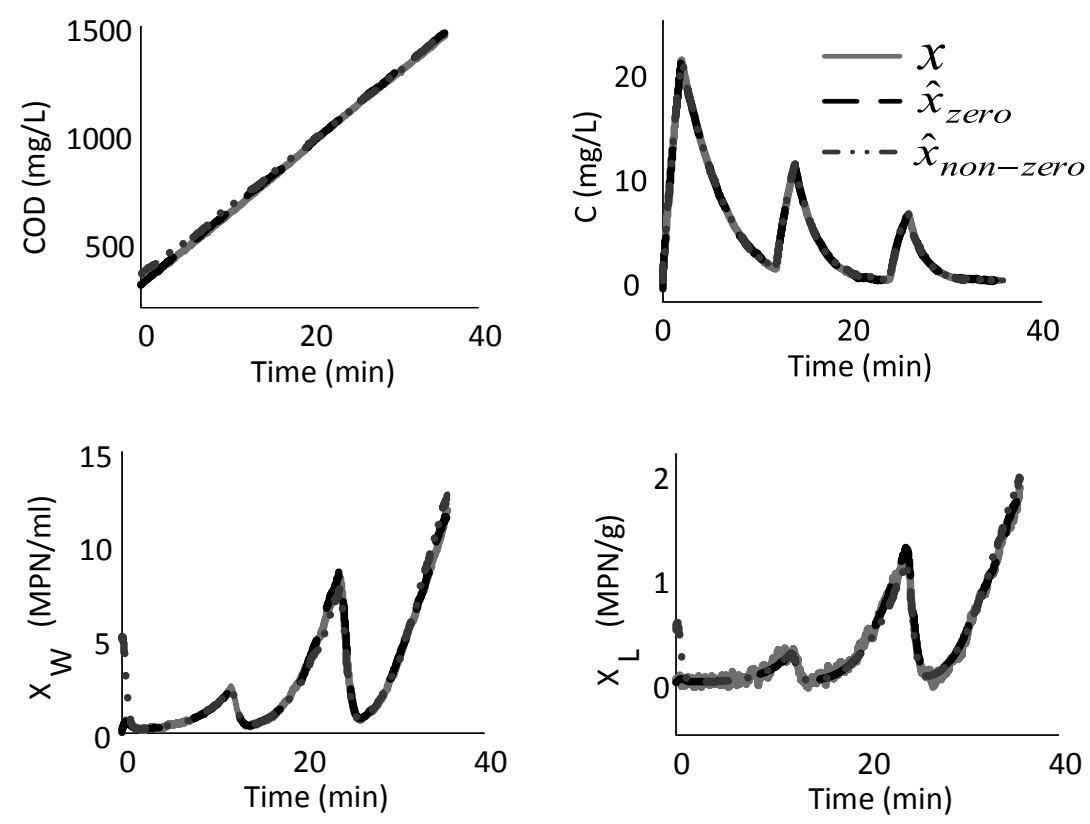

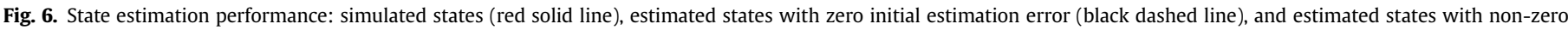
initial estimation error (blue dotted-dashed line).
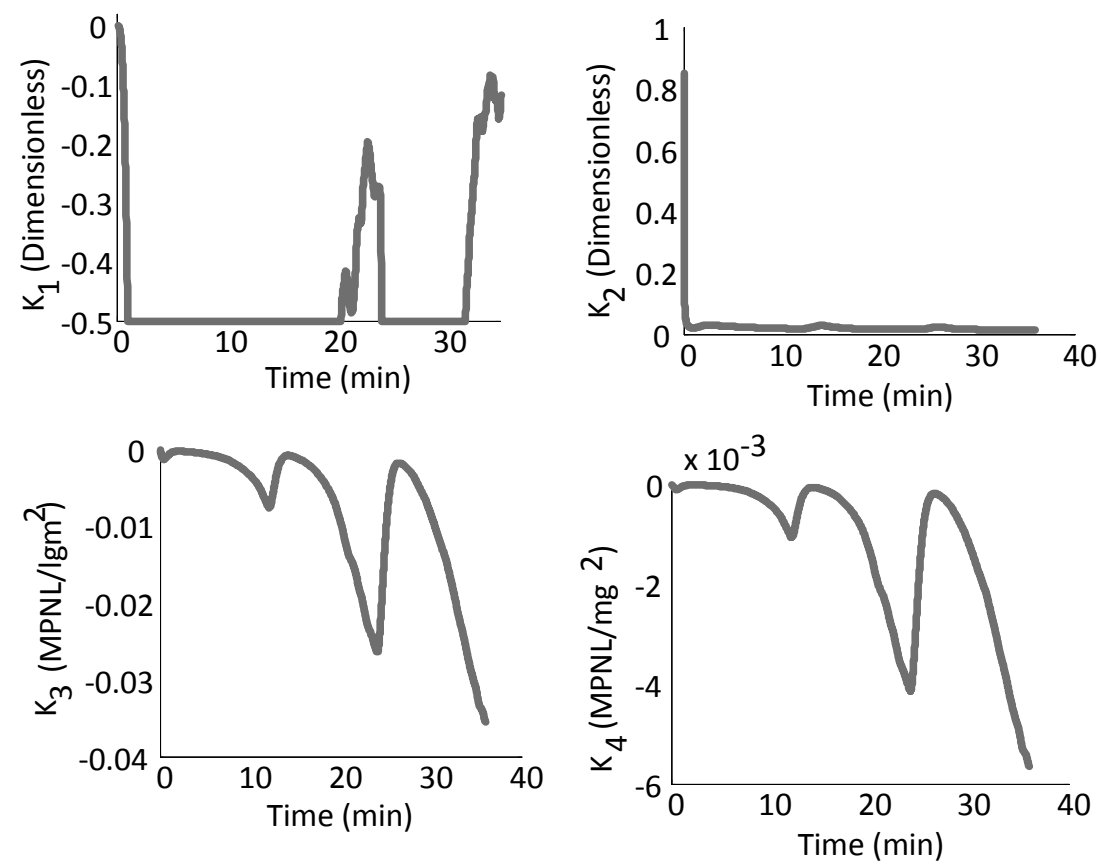

Fig. 7. Kalman filter gains of the HEKF algorithm. A large-magnitude gain indicates that the state estimate is sensitive to the free chlorine measurement.

$S_{e_{j}}=\sqrt{\frac{1}{N_{m c}} \sum_{i=1}^{N_{m c}}\left(x_{j i}-\widehat{x}_{j i}\right)^{2}}$

where $x_{j i}$ and $\widehat{x}_{j i}(j=1, \ldots, 4)$ denote the state and its estimate at the $i^{\text {th }}$ time step.
Fig. 10 shows the average standard deviation of the estimation error for each state over 100 Monte Carlo simulations, each with a 36 min simulation time. The standard deviations are bounded within reasonable ranges: $5 \mathrm{mg} / \mathrm{L}$ for COD, $0.1 \mathrm{mg} / \mathrm{L}$ for FC, 0.1 $\mathrm{MPN} / \mathrm{ml}$ for PC, and $0.025 \mathrm{MPN} / \mathrm{g}$ for P on the lettuce. The state estimates are clustered around the true states and the HEKF provides accurate estimates of the states. 


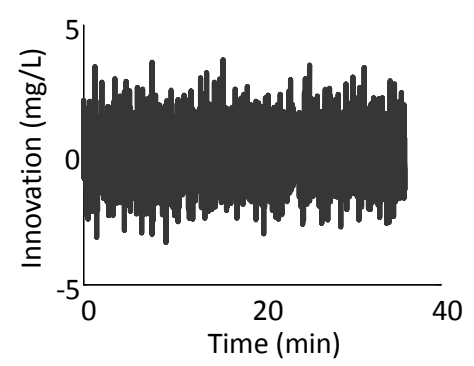

Fig. 8. Innovations of Eq. (9), which is used to update the state estimate in Eq. (8). Note that the innovations appear to be white and zero-mean, which implies the optimality of the filter.

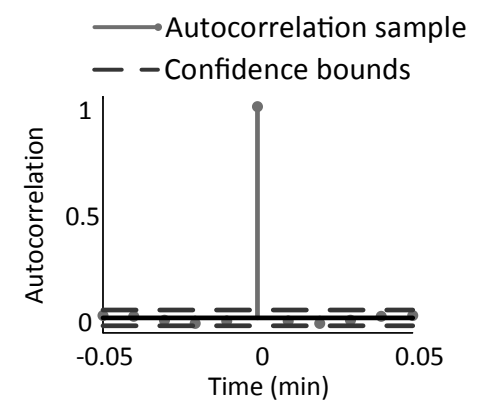

Fig. 9. White noise test of the innovations: autocorrelation of the innovations (red solid circle), and 99\% confidence bounds (blue dashed lines).

\subsection{Sensitivity analysis}

Robustness is a basic requirement for a state estimator. The question addressed here is whether the HEKF and the noise optimization algorithm provide robustness to system parameter deviations (that is, modeling errors) and are thus reliable as a practical method for real-world plant wash systems. A robustness test is performed by varying all of the system parameters, one at a time, and calculating the sensitivity of each state estimation error relative to each perturbed system model parameter. The following equations are proposed to quantify the effect of system model parameters on state estimation errors:

$\bar{S}_{j}^{P_{l_{\delta}}}=\sqrt{\frac{1}{N} \sum_{i=1}^{N}\left(S_{e_{j}}^{P_{l_{\delta}}}(i)\right)^{2}}$

$D_{j}^{P_{I}}=\frac{\left(\frac{\bar{S}_{j}^{P_{l+\delta}}+\bar{S}_{j}^{P_{l-\delta}}}{2}\right)-\bar{S}_{j}^{P_{l_{0}}}}{\bar{S}_{j}^{P_{l_{0}}}} \times 100$

$S E_{j}^{P_{l}}=\frac{D_{j}^{P_{l}}}{\delta \%}$

$S_{e_{j}}^{P_{l_{\delta}}}(i)$ represents the standard deviation of the estimation error of the $j^{\text {th }}$ state at the $i^{\text {th }}$ time step, averaged over $N_{m c}$ Monte Carlo simulations, when the estimator's model of the $l^{\text {th }}$ system parameter has an error of $\delta \%$ relative to its true value while the other system parameters are modeled correctly. $\bar{S}_{j}^{P_{\delta}}$ denotes the mean of
$S_{e_{j}}^{P_{l}}$ over $N$ time steps. $D_{j}^{P_{l}}$ indicates the RMSE percentage change of the estimation error of the $j^{\text {th }}$ state due to a modeling error in the $l^{\text {th }}$ system parameter. $S E_{j}^{P_{l}}$ denotes the sensitivity of the estimation error of the $j^{\text {th }}$ state relative to an error in the $l^{\text {th }}$ system model parameter, where a positive sensitivity means that the estimation error of the $j^{\text {th }}$ state increases, and a negative sensitivity means that the estimation error of the $j^{\text {th }}$ state decreases.

Fig. 11 shows the sensitivity of the error of each state estimate to the error of each system model parameter, which were calculated with a value of $\delta=10$ in Eqs. (19)-(21). The largest sensitivity is that of the $\operatorname{COD}(O)$ estimation error relative to the COD increase rate $\left(K_{0}\right)$; this is because the derivative of the COD state is exactly equal to $K_{0}$, as seen in Eq. (1). The smallest sensitivity is that of the $P$ on the lettuce $\left(X_{L}\right)$ estimation error relative to the decay rate of FC $\left(\gamma_{c}\right)$, which is about 0 ; although $\gamma_{c}$ appears in the derivative of the FC concentration in Eq. (1) and chlorine measurements are used to update the state estimates, the cross-contamination dynamics, and in turn $X_{L}$, are not directly dependent on $\gamma_{c}$.

As seen from Fig. 11, the estimate of COD is also highly sensitive to the FC consumption rate $\beta_{c}$. This is because the first element of the covariance $Q_{s}$ (shown at the end of Section 2), which corresponds to COD process noise, is very large relative to the other elements of $Q_{s}$. This indicates that there is a significant difference between the experimental data and the COD model dynamics of Eq. (1). Conversely, the second element of the covariance matrix is very small, indicating a close match between the experimental data and the FC model dynamics of Eq. (1). These two observations lead to the conclusion that when $\beta_{c}$ is modeled incorrectly the Kalman filter adjusts its estimate of $\operatorname{COD}\left(x_{1}\right)$, but not FC $\left(x_{2}\right)$, to maintain a close match between the experimental data and the $\beta_{c} x_{1} x_{2}$ term in the second dynamic equation of Eq. (1).

Similarly, Fig. 11 shows that COD estimation errors have a relatively high sensitivity to almost all of the model parameters. Again, this is because the first element of the covariance matrix, which corresponds to COD process noise, is very large relative to its other elements. This, in turn, allows the Kalman filter to significantly adjust its estimate of COD to compensate for modeling errors.

Fig. 11 shows that the estimated FC concentration $(C)$ is not very sensitive to modeling errors. Its greatest sensitivity is to the modeled value of FC consumption rate $\left(\beta_{c}\right)$. This is because $\beta_{c}$ appears directly in the derivative of the FC state in Eq. (1).

The estimated PC in the water $\left(X_{W}\right)$ is not very sensitive to modeling errors. Its greatest sensitivity is to the interaction rate of the pathogen via $\mathrm{FC}(\alpha)$. This is because of their direct relationship in the system dynamics of Eq. (1). Note that the estimate of $X_{W}$ has a negative sensitivity to several modeling parameters. This means that the estimate actually improves in the presence of modeling errors. This phenomenon is difficult to explain but is probably due to the highly nonlinear nature of the system dynamics, a characteristic which often precludes the analytical or intuitive explanations of sensitivity relationships. The negative sensitivities indicate that $X_{W}$ can be accurately estimated even without a good knowledge of the system model.

Finally, the estimated $P$ on the lettuce $\left(X_{L}\right)$ is not very sensitive to modeling errors. Its greatest sensitivity is to the pathogen binding rate $\left(\beta_{l w}\right)$. This is because of their direct relationship in the system dynamics.

In summary, the proposed state estimator has good performance even when the system parameters deviate from their modeled values. This analysis indicates that the HEKF and noise statistics optimization algorithm are robust to system parameter deviations and could thus be reliable for real-world plant wash systems. 

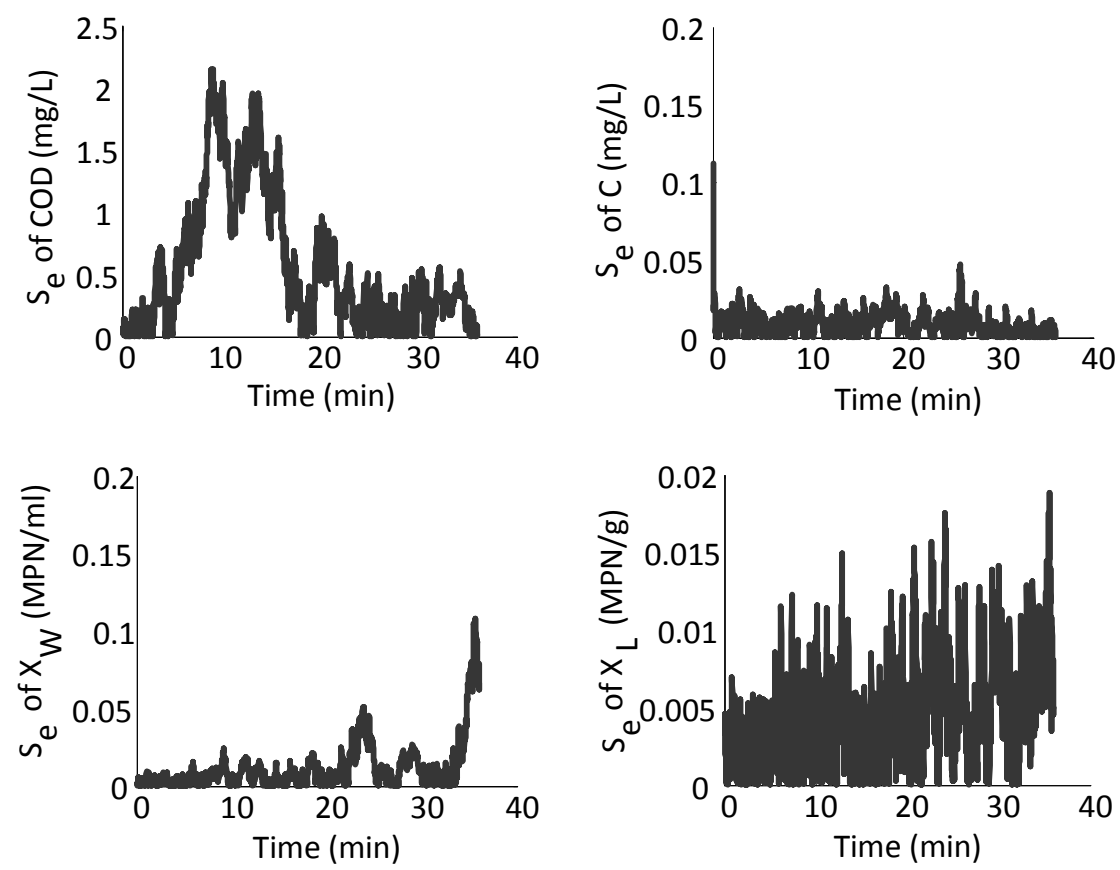

Fig. 10. Standard deviation of the estimation errors for the system states, averaged over 100 Monte Carlo simulations, each with a 36 min simulation time.

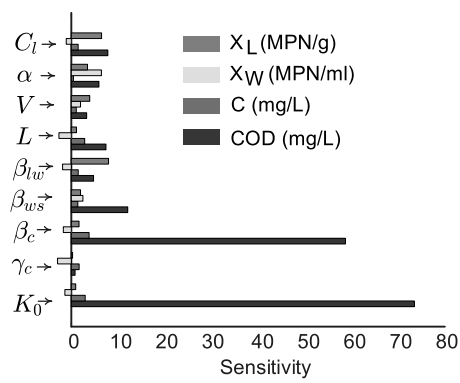

Fig. 11. Sensitivities of the four state estimation errors relative to modeling errors in the nine system parameters.

\section{Conclusion and future work}

An HEKF was designed to estimate the states of a continuoustime wash system using discrete-time FC measurements. PSO was used to optimize the HEKF process and measurement noise covariances so that the innovations satisfied theoretically desired properties. Simulation results showed that PSO decreased the cost function by $34 \%$. RMS estimation errors were $8.24 \mathrm{mg} / \mathrm{L}$ for the COD, $0.09 \mathrm{mg} / \mathrm{L}$ for the FC concentration, $0.19 \mathrm{MPN} / \mathrm{ml}$ for PC in the water wash, and $0.04 \mathrm{MPN} / \mathrm{g}$ for the P on the lettuce in the tank. A sensitivity analysis showed that, as expected, the highest sensitivity was that of the COD estimation error to COD increase rate modeling errors. In general, the estimation errors showed a low sensitivity to modeling errors, confirming the robustness of the estimator.

For future work, a state-based controller for the produce wash system will be designed to control FC concentration, the pathogen concentration in the water wash, and the amount of pathogen on the lettuce during the wash process, all while minimizing the FC input.

The simulation results in this paper can be reproduced with the Matlab source code that is available at http://embeddedlab.csuohio. edu/plant-wash-estimation.

\section{References}

Barrera, M.J., et al., 2012. The effect of different processing parameters on the ef ficacy of commercial post-harvest washing of minimally processed spinach and shredded lettuce. Food Control 25 (2), 745-751.

Bellantoni, J., Dodge, K., 1967. A square root formulation of the Kalman-Schmidt filter. Am. Inst. Aeronautics Astronautics J. 5 (7), 1309-1314.

Beuchat, L.R., et al., 2004. Efficacy of chlorine and a peroxyacetic acid sanitizer in killing Listeria monocytogenes on iceberg and romaine lettuce using simulated commercial processing conditions. J. Food Prot. 67 (6), 1238-1242.

Chen, X., Hung, Y.C., 2016. Predicting chlorine demand of fresh and fresh-cut produce based on produce wash water properties. Postharvest Biol. Technol. 120, $10-15$.

Deborde, M., Von Gunten, U.R.S., 2008. Reactions of chlorine with inorganic and organic compounds during water treatment kinetics and mechanisms: a critical review. Water Res. 42 (1), 13-51.

Eberhart, R.C., Kennedy, J., 1995. A new optimizer using particle swarm theory. In: Proceedings of the Sixth International Symposium on Micro Machine and $\mathrm{Hu}-$ man Science. Nagoya Municipal Industrial Research Institute, pp. 39-43.

Fakoorian, S.A., et al., 2016. Ground reaction force estimation in prosthetic legs with an extended Kalman filter. In: IEEE International Systems Conference, pp. 338-343. Orlando, Florida.

Fakoorian, S.A., et al., 2017. Ground reaction force estimation in prosthetic legs with nonlinear Kalman filtering methods. J. Dyn. Syst. Meas. Control. http://dx.doi. org/10.1115/1.4036546.

Gil, M.I., et al., 2009. Fresh-cut product sanitation and wash water disinfection: problems and solutions. Int. J. Food Microbiol. 134 (2), 37-45.

Gómez-López, V., et al., 2014. Minimum free chlorine residual level required for the inactivation of Escherichia coli 0157: H7 and trihalomethane generation during dynamic washing of fresh-cut spinach. Food Control 42, 132-138.

Hach Chemical Company, 2002. Water Analysis Handbook, Chemical Oxygen Demand, Method 8000, Loveland, CO, pp. 1-8.

Hashemian, N., Armaou, A., 2015. Fast moving horizon estimation of nonlinear processes via carleman linearization. In: American Control Conference pp. 3379-3385. Chicago, IL.

Hashemian, N., Armaou, A., 2016. Simulation, model-reduction, and state estimation of a two-component coagulation process. AIChE J. http://dx.doi.org/ 10.1002 /aic.15146.

Kennedy, J., Eberhart, R.C., 1995. Particle swarm optimization. In: IEEE International Conference on Neural Networks, Perth, Australia, pp. 1942-1948.

Kontoroupi, T., Smyth, A.W., 2016. Online noise identification for joint state and parameter estimation of nonlinear systems. SCE-ASME J. Risk Uncertain. Eng. Syst. Part A Civ. Eng. 2 (3). B4015006-1-B4015006-12.

Kost, O., et al., 2015. Identification of state and measurement noise covariance matrices using nonlinear estimation framework. J. Phys. Conf. Ser. 659 (1-12), 1

Luo, Y., 2007. Fresh-cut produce wash water reuse affects water quality and packaged product quality and microbial growth in romaine lettuce. HortScience 42 (6), 1413-1419.

Luo, Y., et al., 2011. Determination of free chlorine concentrations needed to preven 
Escherichia coli 0157:H7 cross-contamination during fresh-cut produce wash. J. Food Prot. 74 (3), 352-358.

Luo, Y., et al., 2012. A pilot plant scale evaluation of a new process aid for enhancing chlorine efficacy against pathogen survival and cross-contamination during produce wash. Int. J. Food Microbiol. 158 (2), 133-139.

Lynch, M.F., et al., 2009. The growing burden of foodborne outbreaks due to contaminated fresh produce: risks and opportunities. Epidemiol. Infect. 137 (3), 307-315.

Munther, D., et al., 2015. A mathematical model for pathogen cross-contamination dynamics during produce wash. Food Microbiol. 51, 101-107.

Nou, X., Luo, Y., 2010. Whole-leaf wash improves chlorine efficacy for microbial reduction and prevents pathogen cross-contamination during fresh-cut lettuce processing. J. food Sci. 75 (5), M283-M290.

Park, S., et al., 2012. Risk factors for microbial contamination in fruits and vegetables at the preharvest level: a systematic review. J. food Prot. 75 (11) 2055-2081.

Pirovani, M., et al., 2001. Predictive models for available chlorine depletion and total microbial count reduction during washing of fresh-cut spinach. J. Food Sci. 66 (6), 860-864.
Rodríguez, F.P., et al., 2011. A mathematical risk model for Escherichia coli 0157: H7 cross-contamination of lettuce during processing. Food Microbiol. 28 (4), 694-701.

Shen, C., et al., 2013. Dynamic effects of free chlorine concentration, organic load, and exposure time on the inactivation of Salmonella, Escherichia coli 0157: H7, and non-0157 Shiga toxin-producing E. coli. J. Food Prot. 76 (3), 386-393.

Simon, D., 2006. Optimal State Estimation: Kalman, H-infinity, and Nonlinear Approaches. John Wiley \& Sons.

Simon, D., 2013. Evolutionary Optimization Algorithms. John Wiley \& Sons.

Sivapalasingam, S., et al., 2004. Fresh produce: a growing cause of outbreaks of foodborne illness in the United States, 1973 through 1997. J. Food Prot. 67 (10), $2342-2353$.

Van Haute, S., et al., 2013. Physicochemical quality and chemical safety of chlorine as a reconditioning agent and wash water disinfectant for fresh-cut lettuce washing. Appl. Environ. Microbiol. 79 (9), 2850-2861.

Zhou, B., et al., 2014. Development of an algorithm for feed-forward chlorine dosing of lettuce wash operations and correlation of chlorine profile with Escherichia coli 0157: H7 inactivation. J. Food Prot. 77 (4), 558-566. 